\title{
On New Solutions of Classical Yang-Mills Equations with Cylindrical Sources
}

\author{
Alexander S. Rabinowitch \\ Department of Applied Mathematics, Moscow State University of Instrument Construction and Information Sciences, \\ Moscow, Russia \\ E-mail:rabial@dol.ru
}

Received April 27, 2010; revised May 14, 2010; accepted May 17, 2010

\begin{abstract}
Strong fields generated by big electric currents are examined within the framework of the Yang-Mills nonlinear generalization of the Maxwell electrodynamics proposed in our earlier papers. First we consider the case of stationary currents and find a new exact solution to the Yang-Mills equations. Then we study a Yang-Mills field inside a thin circular cylinder with nonstationary plasma and find expressions for field strengths in it. Obtained results are applied to interpret several puzzling natural phenomena.
\end{abstract}

Keywords: Yang-Mills Equations, Su(2) Symmetry, Source Currents, Field Strengths, Lightning, Exploding Wires

\section{Introduction}

As is well known, the Yang-Mills field theory proposed in 1954 is one of the greatest achievements of the XX century, which plays a leading role in modern quantum physics [1-3]. At the same time, the whole area of its applications can concern not only quantum physics but also classical physics [4-7]. To explain this point of view, let us examine powerful fields generated by sources with very big electric charges and currents. Then the following question should be raised. Are the classical Maxwell equations always applicable to such fields?

It is beyond doubt that the Maxwell equations adequately describe a great diversity of electromagnetic fields for which photons are their carriers. At the same time, powerful sources with very big charges and currents may generate not only photons but also $\mathrm{Z}^{0}$ and $\mathrm{W}^{ \pm}$bosons. In such cases, the Maxwell equations may be incorrect since they are applicable to fields for which only photons are the carriers. On the other hand, there are the well-known Yang-Mills equations with $S U(2)$ symmetry which are a nonlinear generalization of the linear Maxwell equations playing a leading role in various models of electroweak interactions caused by photons and $Z^{0}$ and $W^{ \pm}$bosons. For this reason, in [4-7] the classical Yang-Mills equations with $S U(2)$ symmetry are applied in the case of powerful field sources with very big electric charges and currents when $\mathrm{Z}^{0}$ and $\mathrm{W}^{ \pm}$ bosons may be generated, along with photons. These equations can be represented in the form [1-3]

$$
\begin{gathered}
D_{\mu} F^{k, \mu v} \equiv \partial_{\mu} F^{k, \mu v}+g \varepsilon_{k l m} F^{l, \mu v} A_{\mu}^{m}=(4 \pi / c) j^{k, v}, \\
F^{k, \mu v}=\partial^{\mu} A^{k, v}-\partial^{v} A^{k, \mu}-g \varepsilon_{k l m} A^{l, \mu} A^{m, v},
\end{gathered}
$$

where $\mu, v=0,1,2,3 ; k, l, m=1,2,3, A^{k, v}$ and $F^{k, \mu v}$ are potentials and strengths of a Yang-Mills field, respectively, $j^{k, v}$ are three 4-vectors of source current densities, $\varepsilon_{k l m}$ is the antisymmetric tensor, $\varepsilon_{123}=1$, $D_{\mu}$ is the Yang-Mills covariant derivative, $g$ is the constant of electroweak interaction, and $\partial_{\mu} \equiv \partial / \partial x^{\mu}$, where $x^{\mu}$ are orthogonal space-time coordinates of the Minkowski geometry.

It is worth noting that Equations (1-2) have the following well-known consequences [1-3]:

$$
\begin{gathered}
D_{v} D_{\mu} F^{k, \mu v}=0, \\
D_{v} j^{k, v} \equiv \partial_{v} j^{k, v}+g \varepsilon_{k l m} j^{l, v} A_{v}^{m}=0 .
\end{gathered}
$$

Further we will consider the field sources $j^{k, v}$ of the form

$$
j^{1, v}=j^{v}, \quad j^{2, v}=j^{3, v}=0,
$$

where $j^{v}$ is a classical 4-vector of current densities.

Then when the potentials $A^{2, v}=A^{3, v}=0$, the YangMills Equations (1-2) become coinciding with the Maxwell equations for the potentials $\mathrm{A}^{1, v}$. Moreover, from (4) 
with $\mathrm{k}=1$ and from (5) we obtain the differential charge conservation equation

$$
\partial_{v} j^{1, v}=0 .
$$

That is why Equations (1-2) with field sources of Formula (5) can be regarded as a reasonable nonlinear generalization of the classical Maxwell equations. This nonlinear generalization was studied in [4-7], where new classes of spherically symmetric and wave solutions to the considered Yang-Mills equations were obtained. These solutions were applied to interpret puzzling properties of atmospheric electricity, the phenomenon of ball lightning, and some other natural phenomena unexplained within the framework of the linear Maxwell theory [4-7].

It should be noted that from (1)-(6) we come to the identity

$$
D_{v}\left[D_{\mu} F^{k, \mu v}-(4 \pi / c) j^{k, v}\right] \equiv 0 \text { for } k=1
$$

This identity shows that there is a differential relation for the Yang-Mills Equation (1) with the classical sources of Formula (5).

Consider now the classical Yang-Mills Equations (1-2) with cylindrical sources $\mathrm{j}^{\mathrm{k}, v}$ of the following form:

$$
\begin{gathered}
j^{1,0}=j^{0}(\tau, \rho, z), \quad j^{1,3}=j(\tau, \rho, z), \\
j^{1,1}=j^{1,2}=0, \quad j^{2, v}=j^{3, v}=0,
\end{gathered}
$$

where

$$
\tau=x^{0}, \rho=\sqrt{x^{2}+y^{2}}, x=x^{1}, y=x^{2}, z=x^{3} .
$$

Then let us seek the potentials $A^{k, v}$ in the form

$$
\begin{aligned}
& A^{k, 0}=\lambda^{k}(\tau, \rho, z), A^{k, 1} \\
& =x \gamma^{k}(\tau, \rho, z), A^{k, 2} \\
& =y \gamma^{k}(\tau, \rho, z), A^{k, 3}, \\
& =\delta^{k}(\tau, \rho, z)
\end{aligned}
$$

where $\lambda^{k}, \gamma^{k}, \delta^{k}$ are some functions.

Substituting expressions (10) into Formula (2) for the strengths $\mathrm{F}^{\mathrm{k}, \mu v}$ and taking into account the antisymmetry of $\varepsilon_{k l m}$, we find

$$
\begin{aligned}
& F^{k, 01}=x u^{k}, F^{k, 02}=y u^{k}, F^{k, 03}=p^{k}, \\
& F^{k, 12}=0, F^{k, 13}=x h^{k}, F^{k, 23}=y h^{k}, \\
& u^{k}=u^{k}(\tau, \rho, z), p^{k}=p^{k}(\tau, \rho, z), \quad h^{k}=h^{k}(\tau, \rho, z),
\end{aligned}
$$

where the functions $u^{k}, p^{k}$, and $h^{k}$ are as follows:

$$
\begin{aligned}
& u^{k}=\gamma_{\tau}^{k}+\lambda_{\rho}^{k} / \rho-g \varepsilon_{k l m} \lambda^{l} \gamma^{m}, \\
& p^{k}=\delta_{\tau}^{k}+\lambda_{z}^{k}-g \varepsilon_{k l m} \lambda^{l} \delta^{m}, \\
& h^{k}=\gamma_{z}^{k}-\delta_{\rho}^{k} / \rho+g \varepsilon_{k l m} \delta^{l} \gamma^{m} .
\end{aligned}
$$

Here $\gamma_{\tau}^{k} \equiv \partial \gamma^{k} / \partial \tau, \quad \lambda_{z}^{k} \equiv \partial \lambda^{k} / \partial z, \quad \delta_{\rho}^{k} \equiv \partial \delta^{k} / \partial \rho$.

After substituting expressions (8-11) for $\mathrm{j}^{\mathrm{k}, v}, \mathrm{~A}^{\mathrm{k}, v}$, and $\mathrm{F}^{\mathrm{k}, \mu v}$ into the Yang-Mills Equation (1), we obtain

$$
\begin{gathered}
\rho u_{\rho}^{k}+2 u^{k}+p_{z}^{k}-g \varepsilon_{k l m}\left(\rho^{2} u^{l} \gamma^{m}+p^{l} \delta^{m}\right), \\
=-(4 \pi / c) s^{k} j^{0} \\
u_{\tau}^{k}-h_{z}^{k}+g \varepsilon_{k l m}\left(u^{l} \lambda^{m}+h^{l} \delta^{m}\right)=0, \\
p_{\tau}^{k}+\rho h_{\rho}^{k}+2 h^{k}+g \varepsilon_{k l m}\left(p^{l} \lambda^{m}-\rho^{2} h^{l} \gamma^{m}\right)=(4 \pi / c) s^{k} j,
\end{gathered}
$$

where

$$
s^{1}=1, \quad s^{2}=s^{3}=0 .
$$

In the considered case (8), from the three Equations (4) we obtain

$$
\begin{gathered}
j_{\tau}^{0}+j_{z}=0, \\
j^{0} \lambda^{2}-j \delta^{2}=0, \quad j^{0} \lambda^{3}-j \delta^{3}=0 .
\end{gathered}
$$

From (8) we have $j^{2, v}=j^{3, v}=0$. That is why we can choose the following gauge by some rotation about the first axis in the gauge space:

$$
j^{0} \lambda^{1}-j \delta^{1}=0 .
$$

Then using (18), we obtain

$$
j^{0} \lambda^{k}=j \delta^{k}, \quad k=1,2,3
$$

In the second section we study Yang-Mills fields generated by stationary currents flowing in the direction of the axis $z$ and find a particular exact solution to the Yang-Mills Equations (12-16). In the third section we examine these equations in the case of no stationary plasma flowing through a thin circular cylinder and study the Yang-Mills field inside the cylinder. In the fourth section we discuss obtained results and their applications to some puzzling phenomena appearing in lightning and exploding wires.

\section{A Particular Exact Solution to the Yang-Mills Equations in the Case of a Cylindrical Source with Stationary Current}

Let us turn to the considered Yang-Mills Equations (12-16) in the following stationary case:

$$
j=j(\rho), \quad j^{0}=\chi_{0} j(\rho), \quad \chi_{0}=\text { const },
$$

where $j(\rho)$ is some function of $\rho$.

Let us seek the functions $\lambda^{k}, \delta^{k}$, and $\gamma^{k}$ in (10) in the form

$$
\lambda^{k}=\delta^{k}=0, \quad \gamma^{k}=\left(z-\chi_{0} \tau\right) \phi^{k}(\rho)+\psi^{k}(\rho),
$$

where $\phi^{k}$ and $\psi^{k}$ are some functions of $\rho$. 
Then from Formulas (12) and (22) we find

$$
u^{k}=-\chi_{0} \phi^{k}(\rho), \quad h^{k}=\phi^{k}(\rho), \quad p^{k}=0 .
$$

Substituting Formulas (21-23) into Equations (13-15) and using the antisymmetry of $\varepsilon_{k l m}$ and hence the identity $\varepsilon_{k l m} \phi^{l} \phi^{m} \equiv 0$, we come to the following system of equations:

$$
\rho\left(\phi^{k}\right)^{\prime}+2 \phi^{k}-g \rho^{2} \varepsilon_{k l m} \phi^{l} \psi^{m}=(4 \pi / c) s^{k} j, \quad k=1,2,3 .
$$

Therefore, we have got three equations for the six functions $\phi^{k}(\rho)$ and $\psi^{k}(\rho)$.

Taking into account (16), from Equation (24) we find

$$
\begin{gathered}
\rho\left(\phi^{1}\right)^{\prime}+2 \phi^{1}+g \rho^{2}\left(\phi^{3} \psi^{2}-\phi^{2} \psi^{3}\right)=(4 \pi / c) j, \\
\psi^{2}=\frac{\rho\left(\phi^{3}\right)^{\prime}+2 \phi^{3}+g \rho^{2} \phi^{2} \psi^{1}}{g \rho^{2} \phi^{1}}, \\
\psi^{3}=-\frac{\rho\left(\phi^{2}\right)^{\prime}+2 \phi^{2}-g \rho^{2} \phi^{3} \psi^{1}}{g \rho^{2} \phi^{1}} .
\end{gathered}
$$

From (26) we derive

$$
\begin{aligned}
& g \rho^{2}\left(\phi^{3} \psi^{2}-\phi^{2} \psi^{3}\right) \\
& =\left(\phi^{1}\right)^{-1}\left\{\rho\left[\phi^{2}\left(\phi^{2}\right)^{\prime}+\phi^{3}\left(\phi^{3}\right)^{\prime}\right]+2\left[\left(\phi^{2}\right)^{2}+\left(\phi^{3}\right)^{2}\right]\right\} .
\end{aligned}
$$

Substituting (27) into Equation (25), we readily obtain

$$
\phi\left(\rho \phi^{\prime}+2 \phi\right)=(4 \pi / c) j \phi^{1},
$$

where

$$
(\phi)^{2}=\left(\phi^{1}\right)^{2}+\left(\phi^{2}\right)^{2}+\left(\phi^{3}\right)^{2} .
$$

Since in the case (8) under consideration the axes with $k=2,3$ in the gauge space are equivalent, let us choose the relativistic-invariant gauge condition

$$
F^{2, \mu v} F_{\mu v}^{2}=F^{3, \mu v} F_{\mu v}^{3} .
$$

Then we can take the following form for the components $\phi^{k}$ :

$$
\phi^{1}=\phi \cos \xi, \quad \phi^{2}=\phi^{3}=2^{-1 / 2} \phi \sin \xi,
$$

which satisfies (29) and (30), where $\phi=\phi(\rho)$ and $\xi=\xi(\rho)$.

From (28) and (31) we find

$$
\rho \phi^{\prime}+2 \phi=(4 \pi / c) j \cos \xi .
$$

Equation (32) is the only equation for the two unknown functions $\phi(\rho)$ and $\xi(\rho)$. Therefore, in the case under consideration the Yang-Mills equations cannot allow us to uniquely determine the field strengths $F^{k, \mu v}$. To interpret this, let us turn to identity (7). It shows that the considered Yang-Mills Equation (1) with the classical sources of Formula (5) are not independent and there is a differential relation for them.

Therefore, in order to uniquely determine the field strengths $F^{k, \mu v}$, we should find an additional equation. For this purpose, let us represent the Yang-Mills Equation (1) in the form

$$
\partial_{\mu} F^{k, \mu v}=(4 \pi / c) J^{k, v},
$$

where

$$
J^{k, v}=j^{k, v}-(c g / 4 \pi) \varepsilon_{k l m} F^{l, \mu v} A_{\mu}^{m} .
$$

Taking into account (33) and the evident identity $\partial_{v} \partial_{\mu} F^{k, \mu v} \equiv 0$, we find that the components $J^{k, v}$ satisfy the three differential equations of charge conservation

$$
\partial_{v} J^{k, v}=0 \text {. }
$$

In these equations the values $J^{k, v}$ can be interpreted as components of full current densities. As is seen from (34), they are the sum of the source components $j^{k, v}$ and the second addendum which can correspond to charged field quanta.

Using the components $J^{k, v}$ of full current densities and the source current densities $j^{k, v}$, the following additional relativistic-invariant equation was proposed in [4-6] to uniquely determine the field strengths $F^{k, \mu v}$ :

$$
\sum_{k=1}^{3} J^{k, v} J_{v}^{k}=\sum_{k=1}^{3} j^{k, v} j_{v}^{k} .
$$

The expressions on the left and right of this equation are proportional to the interaction energy of the full currents and source currents, respectively, in a small part of a field source. That is why Equation (36) implies the conservation of this energy when charged field quanta are created inside the source $[5,6]$.

Using (33), we can represent Eq. (36) in the form

$$
\sum_{k=1}^{3} \partial_{\alpha} F^{k, \alpha v} \partial_{\beta} F_{v}^{k, \beta}=(4 \pi / c)^{2} \sum_{k=1}^{3} j^{k, v} j_{v}^{k} .
$$

Substituting expressions (8) and (11) into Equation (37), we find

$$
\begin{aligned}
& \sum_{k=1}^{3}\left[\left(\rho u_{\rho}^{k}+2 u^{k}+p_{z}^{k}\right)^{2}-\rho^{2}\left(u_{\tau}^{k}-2 h^{k}+h_{\tau}^{k}\right)^{2}\right]=(4 \pi / c)^{2}\left[\left(j^{0}\right)^{2}-(j)^{2}\right]
\end{aligned}
$$

Using (21) and (23), from Equation (38) we obtain

$$
\sum_{k=1}^{3}\left[\rho\left(\phi^{k}\right)^{\prime}+2 \phi^{k}\right]^{2}=(4 \pi / c)^{2} j^{2} .
$$

Taking into account Formulas (31), Equation (39) can be represented as

$$
\left(\rho \phi^{\prime}+2 \phi\right)^{2}+\left(\rho \phi \xi^{\prime}\right)^{2}=(4 \pi / c)^{2} j^{2} .
$$


From Equations (32) and (40) we obtain

$$
\rho \phi \xi^{\prime}= \pm(4 \pi / c) j \sin \xi .
$$

Equations (32) and (41) give

$$
\rho \phi^{\prime}+2 \phi= \pm \rho \phi \xi^{\prime} \cot \xi \text {. }
$$

Dividing this equation by $\rho \varphi$ and then integrating it, we find

$$
\int\left(\frac{\phi^{\prime}}{\phi}+\frac{2}{\rho}\right) d \rho= \pm \int \cot \xi d \xi .
$$

Equation (43) gives

$$
\ln \left|\rho^{2} \phi\right|= \pm \ln |\sin \xi|+\text { const } .
$$

In order to have the function $\phi(\rho)$ nonsingular, let us choose the sign ' + ' in Equation (41) and hence in Equation (44). Then from Equation (44) we find

$$
\phi=D_{0} \sin \xi / \rho^{2}, \quad D_{0}=\text { const . }
$$

Since we have chosen the sign ' + ' in Equation. (41), from it and Formula (45) we obtain

$$
\xi^{\prime}=(4 \pi / c) j \rho / D_{0} .
$$

From (45) and (46) we have the following nonsingular solution:

$$
\phi=D_{0} \frac{\sin \xi}{\rho^{2}}, \quad \xi=\frac{4 \pi}{c D_{0}} \int_{0}^{\rho} j \rho d \rho, j=j(\rho) .
$$

Formulas (31) and (47) give

$$
\begin{gathered}
\phi^{1}=\frac{2 D}{c} \sin \left(\frac{I}{D}\right) \frac{1}{\rho^{2}}, \quad D=\frac{c D_{0}}{4}, \\
\phi^{2}=\phi^{3}=\frac{\sqrt{2} D}{c}\left[1-\cos \left(\frac{I}{D}\right)\right] \frac{1}{\rho^{2}}, \\
I=2 \pi \int_{0}^{\rho} j \rho d \rho,
\end{gathered}
$$

where $I=I(\rho)$ is the source current in the cylindrical region of radius $\rho$.

From Equations (11), (23), and (48) we find the nonzero strength components

$$
F^{k, 13}, F^{k, 23}
$$

and

$$
F^{k, 01}=-\chi_{0} F^{k, 13}, \quad F^{k, 02}=-\chi_{0} F^{k, 23} .
$$

For the components $F^{k, 13}$ and $F^{k, 23}$ we have

$$
\begin{aligned}
F^{1,13} & =\frac{2 I_{\mathrm{eff}}}{c} \frac{x}{\rho^{2}}, \quad F^{1,23}=\frac{2 I_{\mathrm{eff}}}{c} \frac{y}{\rho^{2}}, \\
I_{\mathrm{eff}} & =D \sin \left(\frac{I}{D}\right), \quad I=I(\rho),
\end{aligned}
$$

$$
\begin{aligned}
& F^{2,13}=F^{3,13}=\frac{\sqrt{2} D}{c}\left[1-\cos \left(\frac{I}{D}\right)\right] \frac{x}{\rho^{2}}, \\
& F^{2,23}=F^{3,23}=\frac{\sqrt{2} D}{c}\left[1-\cos \left(\frac{I}{D}\right)\right] \frac{y}{\rho^{2}},
\end{aligned}
$$

where $D$ is some constant.

Below we use the terms 'actual' and 'effective' for the currents $I$ and $I_{\text {eff }}=D \sin (I / D)$, respectively.

It should be noted that when $|I|<<|D|$, the effective current $I_{\text {eff }}$ practically coincides with the actual current $I$ and we have the Maxwell field expressions for the strength components $F^{1,13}$ and $F^{1,23}$. The value $|D|$ should be a sufficiently large constant. Then Formula (50) can be regarded as a nonlinear generalization of the corresponding Maxwell field expressions for the strengths components $F^{1,13}$ and $F^{1,23}$ when the actual current $I$ is sufficiently large.

Formula (50) describe a nonlinear effect of field saturation. Namely, let the absolute value of the actual current $I$ be increasing from zero. Then when it reaches the value $\pi|D|$, the strengths components $F^{1,13}$ and $F^{1,23}$ become equal to zero and after that they change theirs signs.

This property could be applied to give a new interpretation for the unusual phenomenon of bipolar lightning that actually changes its polarity (positive becoming negative or vice versa) [8].

It is also interesting to note that puzzling data for lightning were recently obtained by the Fermi Gammaray Space Telescope which could be explained by Formula (50). Namely, some of lightning storms had the surprising sign of positrons, and the conclusion was made that the normal orientation for an electromagnetic field associated with a lightning storm somehow reversed [9].

To explain these data, let us note that as follows from (50), the sign of the effective current $I_{\text {eff }}$ can differ from the sign of the actual current $I$ when the latter is sufficiently large.

\section{Yang-Mills Fields Inside Thin Circular Cylinders With Nonstationary Plasma}

Consider now a nonstationary thin cylindrical source of Formula (8) and let us assume that the matter inside it is in the plasma state.

Besides, let the functions $j^{0}$ and $j$ in (8) have the following form inside the thin source:

$$
j^{0}=j^{0}(\tau, z), \quad j=j(\tau, z), \quad 0 \leq \rho<\rho_{0}
$$


where $\rho_{0}$ is its radius.

Our objective is to describe the Yang-Mills field inside the source and for this purpose let us apply Eqs. (12)-(16). In the considered case we seek the functions $\lambda^{k}, \gamma^{k}$, and $\delta^{k}$, describing the field potentials $A^{k, v}$, in the following special form:

$$
\gamma^{k}=0, \lambda^{k}=\lambda^{k}(\tau, z), \delta^{k}=\delta^{k}(\tau, z), 0 \leq \rho<\rho_{0}
$$

Then substituting (52) into Formula (12) and using (20) and the identity $\varepsilon_{k l m} \lambda^{l} \lambda^{m} \equiv 0$, since $\varepsilon_{k l m}$ are antisymmetric, we find

$$
\begin{gathered}
u^{k}=0, \quad h^{k}=0, \\
p^{k}=\delta_{\tau}^{k}+\lambda_{z}^{k}, \quad j^{0} \lambda^{k}=j \delta^{k}
\end{gathered}
$$

It should be noted that in the examined case, as follows from (11) and (53), the values $F^{k, 03}=-F^{k, 30}$ are the only nonzero field strengths. That is why the chosen Formula (52) for the field potentials (10) provides the absence of currents in the directions orthogonal to the axis $z$ of the considered cylindrical source in the plasma state, in accordance with the used Formula (8) for the source.

Substituting now expressions (52) and (53) into Eqs. (13)-(15), we obtain

$$
\begin{aligned}
& p_{z}^{k}-g \varepsilon_{k l m} p^{l} \delta^{m}=-(4 \pi / c) s^{k} j^{0}, \\
& p_{\tau}^{k}+g \varepsilon_{k l m} p^{l} \lambda^{m}=(4 \pi / c) s^{k} j
\end{aligned}
$$

where, as indicated in (16), $s^{1}=1, s^{2}=s^{3}=0$.

Let us multiply Equations (55) and (56) by $j$ and $j^{0}$, respectively, and add the products. Then using (20): $j^{0} \lambda^{m}-j \delta^{m}=0$, we obtain

$$
j^{0} p_{\tau}^{k}+j p_{z}^{k}=0 .
$$

To find solutions $p^{k}(\tau, z)$ to Equation (57), let us introduce the function

$$
q=\int_{0}^{\tau} j(\tau, z) d \tau-\int_{0}^{z} j^{0}(0, z) d z .
$$

Consider its partial derivatives. Using expression (58) and Equation (17): $j_{z}=-j_{\tau}^{0}$, we obtain

$$
\begin{gathered}
q_{\tau}=j(\tau, z), \\
q_{z}=\int_{0}^{\tau} j_{z}(\tau, z) d \tau-j^{0}(0, z) . \\
=-\int_{0}^{\tau} j_{\tau}^{0}(\tau, z) d \tau-j^{0}(0, z)=-j^{0}(\tau, z)
\end{gathered}
$$

From Formulas (59) and (60) we find

$$
j^{0} q_{\tau}+j q_{z}=0 .
$$

Taking this into account, we come to the following solutions to the partial differential Equation (57) of the first order for $p^{k}(\tau, z)$ :

$$
p^{k}=p^{k}(q)
$$

where $p^{k}(q)$ are arbitrary differentiable functions.

Indeed, substituting (62) into Equation (57) and taking into account equality (61), we find

$$
j^{0} p_{\tau}^{k}+j p_{z}^{k}=\left(d p^{k} / d q\right)\left(j^{0} q_{\tau}+j q_{z}\right)=0,
$$

and hence, Equation (57) are satisfied.

Thus, as follows from (11), (62), and the first term in (58), the nonzero field strengths $F^{k, 03}$ inside the cylindrical source under consideration depend on all charge passing through unit area of a cross section of the cylindrical source from beginning of the current flow.

Let us turn to Equation (54) and seek the functions $\delta^{k}$ and $\lambda^{k}$, satisfying them, in the form

$$
\begin{aligned}
\delta^{k} & =j^{0}\left[b^{k}(q)+\vartheta(\tau, z) p^{k}(q)\right], \\
\lambda^{k} & =j\left[b^{k}(q)+\vartheta(\tau, z) p^{k}(q)\right],
\end{aligned}
$$

where $b^{k}(q)$ are arbitrary differentiable function and $\vartheta(\tau, z)$ is some differentiable function.

Then substituting expressions (64) into Equation (54) and taking into account equality (17): $j_{\tau}^{0}+j_{z}=0$, we come to the equations

$$
\begin{aligned}
p^{k}= & {\left[d b^{k} / d q+\vartheta d p^{k} / d q\right]\left(j^{0} q_{\tau} .\right.} \\
& \left.+j q_{z}\right)+\left(j^{0} \vartheta_{\tau}+j \vartheta_{z}\right) p^{k}
\end{aligned}
$$

Using Formula (61), from (65) we find

$$
j^{0} \vartheta_{\tau}+j \vartheta_{z}=1
$$

When $j^{0}=0$, from (17) and (66) we have $j=j(\tau)$ and $\vartheta=z / j(\tau)+\vartheta_{0}(\tau)$, where $\vartheta_{0}(\tau)$ is an arbitrary function.

Consider the case $j^{0} \neq 0$. Then in order to solve Eq. (66), it is convenient to choose the variable $q$ instead of the variable $z$ and put

$$
\vartheta=\varphi(\tau, q), \quad q=q(\tau, z) .
$$
find

Indeed, using (67) and Formulas (59) and (60), we

$$
\vartheta_{\tau}=\varphi_{\tau}+j \varphi_{q}, \quad \vartheta_{z}=-j^{0} \varphi_{q},
$$

and substituting (68) into Equation (66), we derive

$$
j^{0} \varphi_{\tau}=1 .
$$

Therefore, we obtain

$$
\varphi(\tau, q)=\int d \tau / j^{0}(\tau, q),
$$

where $j^{0}$ is represented as a function of $\tau$ and $q$.

Let us now substitute Formulas (62) and (64) into Equations (55) and (56). Then using Formulas (59) and (60) and the evident identity, we find that Equations (55) and (56) give the same equations of the following form: 


$$
\begin{gathered}
d p^{k} / d q+g \varepsilon_{k l m} p^{l} b^{m}=(4 \pi / c) s^{k}, \quad k=1,2,3, \\
p^{k}=p^{k}(q), \quad b^{k}=b^{k}(q), \quad s^{1}=1, \quad s^{2}=s^{3}=0 .
\end{gathered}
$$

Multiplying Equation (71) by $2 p^{k}$ and summing the products over, taking into account the antisymmetry of, we obtain

$$
\frac{d}{d q} \sum_{k=1}^{3}\left(p^{k}\right)^{2}=(8 \pi / c) p^{1} .
$$

Besides this equation, from the second and third equations in (71) we also find relations of the functions $b^{2}(q)$ and $b^{3}(q)$ to the functions $b^{1}(q)$ and $p^{k}(q)$.

Let us put

$$
\begin{aligned}
& p^{1}=(4 \pi / c) \beta(q) \cos \zeta(q), \\
& p^{2}=p^{3}=(4 \pi / c) 2^{-1 / 2} \beta(q) \sin \zeta(q),
\end{aligned}
$$

where condition (30) is taken into account, and $\beta(q)$ and $\zeta(q)$ are some functions.

Then substituting expressions (73) into Equation (72), we find

$$
\beta^{\prime}=\cos \zeta .
$$

Let us now turn to Equation (38). From it and (53) we have

$$
\sum_{k=1}^{3}\left[\left(p_{z}^{k}\right)^{2}-\left(p_{\tau}^{k}\right)^{2}\right]=(4 \pi / c)^{2}\left[\left(j^{0}\right)^{2}-(j)^{2}\right] .
$$

Using Formulas (59), (60), and (62), from Equaion (75) we find

$$
\sum_{k=1}^{3}\left(d p^{k} / d q\right)^{2}=(4 \pi / c)^{2}
$$
tain

Substituting Formula (73) into Equation (76), we ob-

$$
\left(\beta^{\prime}\right)^{2}+\left(\beta \zeta^{\prime}\right)^{2}=1,
$$

where $\beta=\beta(q)$ and $\zeta=\zeta(q)$.

Substituting now expression (74) for $\beta^{\prime}$ into Equation (77), we find

$$
\beta \zeta^{\prime}= \pm \sin \zeta
$$

Equations (74) and (78) give

$$
\beta^{\prime} / \beta= \pm \cot \zeta \zeta^{\prime} \text {. }
$$

Let us integrate Equation (79) and choose the sign ' + ' in it and hence in (78), in order to have its nonsingular solution. Then we obtain

$$
\beta=B_{0} \sin \zeta,
$$

where $B_{0}$ is some constant.

Substituting expression (80) into Equation (78) and taking into account that the sign ' + ' has been chosen in it, we find

$$
\zeta^{\prime}=1 / B_{0}, \quad \zeta=q / B_{0}+\zeta_{0},
$$

where $\zeta_{0}$ is some constant.

From Formulas (80) and (81) we obtain

$$
\beta=B_{0} \sin \left(q / B_{0}+\zeta_{0}\right),
$$

where $q$ is defined by Formula (58).

Using Formulas (11), (58), (73), and (82), we find

$$
\begin{aligned}
& F^{1,03}=(2 \pi / c) B_{0} \sin \left(2 q / B_{0}+\eta_{0}\right), \\
& F^{2,03}=F^{3,03}=(\sqrt{2} \pi / c) B_{0}\left[1-\cos \left(2 q / B_{0}+\eta_{0}\right)\right], \\
& \eta_{0}=2 \zeta_{0}, \quad q=\int_{0}^{\tau} j(\tau, z) d \tau-\int_{0}^{z} j^{0}(0, z) d z .
\end{aligned}
$$

As follows from (83), when the value $2 q=-B_{0}\left(\eta_{0}\right.$ $+\pi n)$, where $n$ is an integer, the strength component $F^{1,03}$ is zero and when $n$ is an even integer, $F^{k, 03}=0, k=1,2,3$.

Let us apply obtained results to the puzzling phenomenon of current pause which takes place in exploding wires [10]. The phenomenon proceeds in three stages. At the instant of closure of the circuit, sufficiently large current flows through the wire and causes its explosion. Then in some time the current flow ceases and the period of current pause begins. After a certain period of time the current pause can end and the current flow can continue.

The origin of the current pause is not well understood within the framework of the Maxwell electrodynamics $[10,11]$. That is why let us apply its nonlinear generalization based on the Yang-Mills equations which we have studied. For this purpose, let us turn to Formula (83) and apply them to an exploding wire. As follows from Formula (83), after some period of time the strength component $F^{1,03}$ becomes zero. At this moment the current in the wire should cease. Therefore, Formula (83) allow one to interpret the origin of current pause in exploding wires. The pause could end and the current flow could continue after some redistribution of charges in exploding wires.

\section{Conclusions}

We have studied classical Yang-Mills fields with $S U(2)$ symmetry generated by charged circular cylinders with currents. Our objective was to find solutions to the nonlinear Yang-Mills equations that could generalize the corresponding solutions to the linear Maxwell equations for sufficiently powerful sources.

We considered two cases. In the first of them we studied a Yang-Mills field generated by a stationary current flowing through a circular cylinder. In this case we found a particular exact solution to the Yang-Mills equations. In the obtained solution the strength components $F^{1,13}$ 
and $F^{1,23}$ have the form $F^{1,13}=\left(2 I_{\text {eff }} / c\right) x / \rho^{2}, F^{1,23}=$ $\left(2 I_{\text {eff }} / c\right) y / \rho^{2}, I_{\text {eff }}=D \sin (I / D)$, where $I=I(\rho)$ and $I_{\text {eff }}=I_{\text {eff }}(\rho)$ are the actual and effective currents in the cylindrical region of radius $\rho$, respectively, and $D$ is a sufficiently large constant. When the actual current $I$ is not large and $|I / D|<<1$, the effective current $I_{\text {eff }}$ is very close to the actual current $I$ and the found expressions for $F^{1,13}$ and $F^{1,23}$ are practically coinciding with the corresponding Maxwell field expressions. At the same time, when the actual current $I$ is sufficiently large, the effective current $I_{\text {eff }}$ can substantially differ from the actual current $I$ and, moreover, the values $I$ and $I_{\text {eff }}$ can have different signs. Using this result, we gave a new interpretation for the phenomenon of bipolar lightning and explained the puzzling inversion of the normal orientation for electromagnetic fields associated with some lightning storms which was recently detected by the Fermi Gamma-ray Space Telescope.

In the second case we considered a Yang-Mills field inside a thin circular cylinder with nonstationary plasma. We sought field potentials in Formula (52) and came to the partial differential Equations (54-56). Solving these equations, we found expressions for the field strengths inside the cylindrical source under consideration. It was shown that the strengths could depend on all charge passing through unit area of a cross section of the cylindrical source from beginning of the current flow. The obtained Formula (83) shows that the field strengths inside the cylindrical source can become zero after some period of time. This property of the found solution was above used to explain the puzzling phenomenon of current pause in exploding wires.

\section{References}

[1] L. H. Ryder, "Quantum Field Theory", Cambridge University Press, Cambridge, 1987.

[2] L. D. Faddeev and A. A. Slavnov, "Gauge Fields: Introduction to Quantum Theory," Benjamin-Cummings Publishing, 1990.

[3] P. Frampton, "Gauge Field Theories," Wiley-VCH, 2008.

[4] A. S. Rabinowitch, Russian Journal of Mathematical Physics, Vol. 12, No. 3, 2005, pp. 379-385.

[5] A. S. Rabinowitch, Russian Journal of Mathematical Physics, Vol. 15, 2008, pp. 389-394.

[6] A. S. Rabinowitch, Physics Letters B, Vol. 664, 2008, pp. 295-300.

[7] A. S. Rabinowitch, "Nonlinear Physical Fields and Anomalous Phenomena," Nova Science Publishers, New York, 2009.

[8] V. A. Rakov and M. A. Uman, "Lightning: Physics and Effects," Cambridge University Press, Cambridge, 2003.

[9] R. Cowen, "Signature of Antimatter Detected in Lightening," Science News, Vol. 176, No.12, December 2009, p. 9.

[10] W. G. Chace and H. K. Moore (Eds.), "Exploding Wires," Plenum Press, New York, 1962.

[11] L. I. Urutskoev, V. I. Liksonov and V. G. Tsinoev, "Observation of Transformation of Chemical Elements during Electric Discharge," Applied Physics Reports/ Prikladnaya Fizika, Vol. 4, 2000, pp. 83-100. 